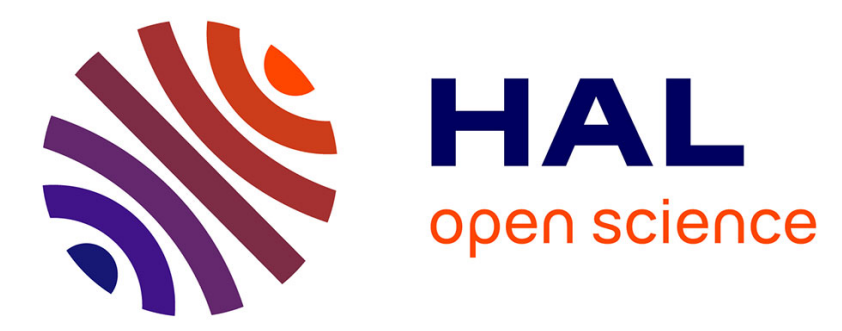

\title{
Palynologie et histoire des activités humaines en milieu montagnard. Bilan provisoire des recherches et nouvelles orientations méthodologiques sur le versant nord des Pyrénées
}

\author{
Didier Galop, Florence Mazier, J.A. Lopez-Saez, Boris Vannière
}

\section{To cite this version:}

Didier Galop, Florence Mazier, J.A. Lopez-Saez, Boris Vannière. Palynologie et histoire des activités humaines en milieu montagnard. Bilan provisoire des recherches et nouvelles orientations méthodologiques sur le versant nord des Pyrénées. Archéologie du Midi Médiéval, 2003, 21, pp.159170. halshs-00958866

\section{HAL Id: halshs-00958866 \\ https://shs.hal.science/halshs-00958866}

Submitted on 13 Mar 2014

HAL is a multi-disciplinary open access archive for the deposit and dissemination of scientific research documents, whether they are published or not. The documents may come from teaching and research institutions in France or abroad, or from public or private research centers.
L'archive ouverte pluridisciplinaire HAL, est destinée au dépôt et à la diffusion de documents scientifiques de niveau recherche, publiés ou non, émanant des établissements d'enseignement et de recherche français ou étrangers, des laboratoires publics ou privés. 


\title{
Palynologie et histoire des activités humaines
} en milieu montagnard. Bilan provisoire des recherches et nouvelles orientations
méthodologiques sur le versant nord des Pyrénées

\author{
Didier GALOP*, Florence MAZIER*, \\ José-Antonio LOPEZ-SAEZ * et Boris VANNIÈRE*
}

\begin{abstract}
Dans l'étude des relations environnement / sociétés en milieu montagnard, la palynologie se positionne aujourd'hui comme une discipline incontournable offrant la possibilité d'aborder la longue durée des processus d'anthropisation. Un examen critique de l'évolution de cette discipline par rapport à la problématique de l'anthropisation peut être mené à partir de l'exemple pyrénéen. S'il ressort que les acquis sont nombreux, ils ne s'accordent pas toutefois systématiquement aux nouvelles demandes formulées par un contexte scientifique résolument orienté vers l'interdisciplinarité. La variabilité des échelles d'analyses temporelles et spatiales constitue un écueil important, mais celui-ci semble pouvoir être contourné par le développement de nouvelles approches paléoécologiques reposant sur des analyses intégrées ainsi que sur la mise en place de référentiels fonctionnels. Nous présentons ici brièvement les résultats de quelques tentatives menées sur le territoire pyrénéen dans le cadre de différents programmes de recherches.

In the study of the environment / societies relationship in the mountain milieu, palynology is now positioned as an unavoidable discipline, offering the possibility of approaching the long period of the anthropisation process. A critical examination of the development of this discipline in relation to the problem of anthropisation can be carried out on the basis of the Pyrenean example. While the acquired elements are numerous, they are not systematically in phase with the new demands required by a scientific context that is resolutely turned towards an interdisciplinary approach. The variability of the scales of temporal and spatial analysis constitutes a major stumbling block, but it seems that this can be overcome by the development of new paleoecological approaches based on integrated analysis, as well as setting up functional references. Here, we briefly present the results of a few attempts carried out in the Pyrenees in the context of different research programmes.
\end{abstract}

Mots-clés : Environnement/sociétés ; anthropisation ; milieu montagnard ; Pyrénées ; palynologie ; micro-fossiles non polliniques ; référentiels fonctionnels ; interdisciplinarité.

Key-words: Environment/societies, anthropisation, mountain milieu, Pyrenees, palynology, non-pollen micro-fossils, functional references, interdisciplinary approach.

Dans le prolongement des travaux sur l'histoire de l'environnement Pyrénéen initiés à la fin des années quatre-vingts à l'initiative des géographes toulousains et des recherches réalisées sur l'estivage en haute Cerdagne (Rendu et al. 1995), nous assistons actuellement à une véritable explosion des investigations sur les relations environnement/ sociétés sur l'ensemble des massifs montagnards de l'hexagone. Désormais, la montagne n'est plus un terrain de recherche marginal et comme le souligne Jean Guilaine, il y a, plus que jamais, " un regard attentif à porter sur la montagne »(Guilaine 1995).

Si les terrains sont différents, certaines constantes réunissent ces recherches. On retiendra surtout une volonté commune d'aborder la longue durée plurimillénaire des processus de socialisation des milieux montagnards, ainsi que, méthodologiquement, la

\footnotetext{
* Laboratoire de Chrono-Ecologie, UMR 6565-CNRS ; 16, route de Gray, F-25030 Besançon Cedex, France.

** Laboratorio de Arqueobotanica, CSIC, Duque de Medinaceli 8, SP-28014 Madrid, Espagne.

Correspondance : didier.galop@univ-fcomte.fr
} 
systématisation des démarches interdisciplinaires qui allient sciences sociales et disciplines de l'environnement, et du paléoenvironnement en particulier. Dans ce concert disciplinaire, force est de constater que les recherches palynologiques occupent une place importante, au point qu'elles apparaissent comme incontournables en se plaçant au centre des dispositifs méthodologiques.

Pour expliquer cette tendance, on pourrait en premier lieu, évoquer l'antériorité des recherches palynologiques sur les terrains montagnards. En effet, bien avant que la montagne devienne le support de recherches pluridisciplinaires elle était le terrain de prédilection des palynologues qui, préoccupés par les dynamiques postglaciaires de la végétation, y trouvaient des sites sédimentaires de choix permettant d'aborder cette problématique que ce soit dans les Pyrénées, mais aussi dans le Massif central, les Alpes ou le Jura. Aussi, une histoire de l'environnement montagnard était-elle préalablement écrite par de nombreuses études palynologiques monographiques. Autant de recherches qui ne pouvaient qu'être intégrées dans les investigations futures.

Ensuite, une autre raison nous paraît reposer sur les atouts propres à la palynologie qui, débarrassée de ses appréhensions relatives aux problèmes de l'anthropisation, offre par l'étude d'enregistrements sédimentaires continus une dimension fondamentale : celle de la longue durée. Longue durée des dynamiques du milieu végétal, mais aussi longue durée des manifestations anthropiques. Aussi, les séquences polliniques constituent-elles de véritables colonnes vertébrales diachroniques sur lesquelles viennent s'articuler les données fragmentaires et parfois désynchronisées des autres disciplines qui y trouvent un lien fédérateur et une trame chronologique, mais aussi un complément d'information lorsque leurs sources documentaires classiques disparaissent. À la lecture de ces propos, très certainement « palynocentrés », on pourrait voir dans la palynologie une sorte de panacée. Ce n'est pas le cas, au contraire. Bien que les atouts de cette discipline soient réels, les collaborations et dialogues entretenus avec les sciences sociales confrontent cette discipline avec ses propres limites et imposent que de nouvelles démarches et de nouvelles orientations méthodologiques soient empruntées. L'évolution récente des travaux palynologiques dans les Pyrénées illustre ces propos. En effet, sur ce massif, l'approche palynologique de l'anthropisation qui remonte au début des années 80 bénéficie d'une histoire suffisamment longue pour qu'un bilan critique puisse en être dressé et pour que s'esquissent de nouvelles pistes de recherches. On déclinera cette histoire -qui ne concerne pas seulement le massif pyrénéen- en trois temps : le temps de «l'impact de l'homme sur la végétation »; celui des « dynamiques rythmiques de l'anthropisation » et enfin le temps présent, que nous nommerons le temps « de l'étude des pratiques ».

\section{DE « L'IMPACT DE L'HOMME SUR LA VÉGÉTATION » À L'ÉTUDE DES DYNAMIQUES RYTHMIQUES DE L'ANTHROPISATION}

Longtemps centrées sur les dynamiques tardi- et postglaciaire de la végétation pyrénéenne, les recherches palynologiques réalisées à partir de la deuxième moitié des années soixante-dix, ne prenaient en compte le facteur anthropique qu'en tant qu'ultime avatar d'une longue histoire essentiellement dominée par des déterminants naturels. Aborder l'anthropisation revenait principalement à décrire l'influence de l'homme sur la végétation et éventuellement à en préciser la chronologie (Jalut 1974 et 1984 ; Reille et Andrieu 1994). Cette position est contemporaine d'un relatif isolement de la recherche palynologique qui s'exerçait de manière autonome et unilatérale autour de problématiques principalement paléoclimatiques.

La rupture avec cette tendance et l'amorce du deuxième temps dans l'approche palynologique de l'anthropisation des Pyrénées, s'effectuent à la fin des années quatre-vingts dans le cadre des programmes PIREN du CNRS. Elles suivaient avec quelques années de retard il est vrai, l'exemple des palynologues d'Europe du nord. Dans ce contexte, le programme « forêt charbonnée » fut déterminant (CIMA URA 366 CNRS, coord. Métailié et Jalut) : il inaugurait le développement d'études polliniques ciblées sur l'histoire des activités humaines y compris pour les périodes les plus récentes jusqu'alors rarement étudiées (Galop 1992). Les travaux se sont par la suite multipliés en différents points du massif, avec pour ambition de saisir les grandes étapes, les rythmes et les conséquences phytogéographiques de l'anthropisation de la chaîne pyrénéenne (Galop 1998 ; Belet 2001 ; Aubert 2001). D'un point de vue méthodologique, qu'ils aient été ou non articulés avec des investigations archéologiques ou historiques, ces travaux reposaient sur un dispositif qui donnait la priorité non plus au site sédimentaire mais davantage à l'aire d'étude (Galop 1998). Aux longues séquences, on préférait des enregistrements plus modestes, à caractère local, situés dans des secteurs représentatifs en termes d'anthropisation (Kvamme 1998). Toutefois, ceci supposait en amont une bonne connaissance des terrains et de leur histoire. Aussi, la multiplication des sondages dans une même zone d'étude, permettait-elle par un emboîtement des échelles d'analyses le passage d'informations locales à des données à valeurs plus régionales. De proche en proche, et par une démarche comparatiste, il devenait alors possible d'envisager une synthèse à l'échelle d'une partie du massif Pyrénéen. Cela fut tenté pour les Pyrénées de l'Est (Galop 1998) et reste en cours de réalisation dans la partie occidentale de la chaîne.

Concernant maintenant des aspects strictement palynologiques, l'analyse de l'anthropisation repose sur la prise en compte d'une série d'indices désormais classiques. En premier lieu, ce sont les déboisements liés 


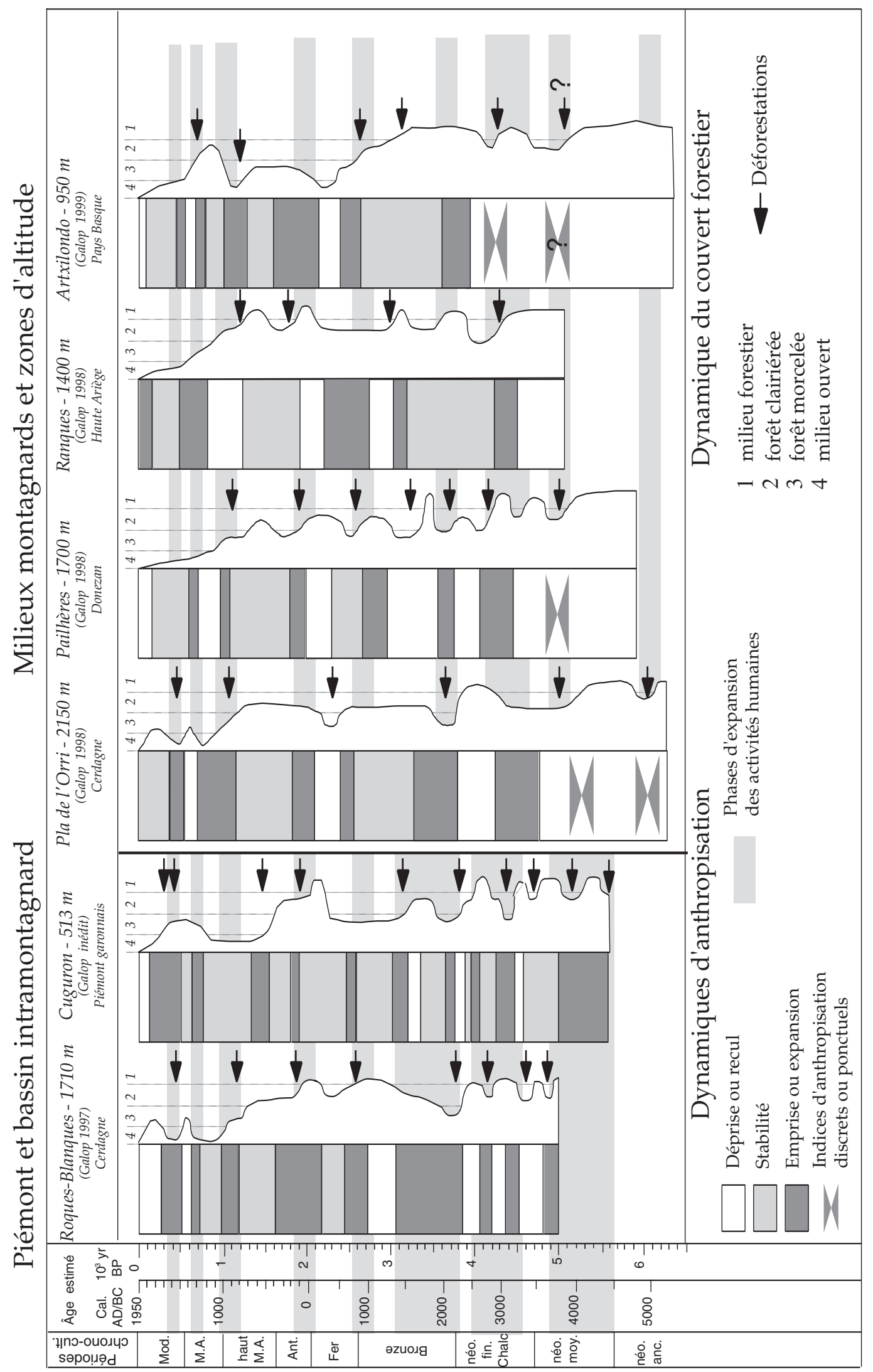

Fig. 1 - Dynamiques rythmiques de l'anthropisation sur le versant nord des Pyrénées (d'après Galop 2001)

Les colonnes figurant les différentes phases de l'anthropisation sont établies à partir de la variation des indices polliniques d'activité humaine dans chacune des séquences polliniques considérées. Les chronologies proposées sont réalisées sur la base d'une estimation des âges par interpolation des datations radiocarbones. L'estimation de l'évolution du couvert forestier ne représente qu'une appréciation et ne saurait avoir une réelle signification quantitative. 
à la conquête de terre, qui en constituent la manifestation la plus évidente : ils sont mis en relief dans les diagrammes palynologiques par le recul des fréquences des essences forestières ainsi que par l'élévation des herbacées héliophiles et de certains arbres recolonisateurs des milieux ouverts (genévrier, bouleau, noisetier...). Cependant, de tels événements peuvent avoir des causes multiples qui n'impliquent pas systématiquement une intervention humaine (aléas météorologiques, incendies naturels, etc.) et d'autres indices sont nécessaires. C'est à ce niveau de la démarche qu'intervient l'utilisation désormais classique des taxons indicateurs de l'anthropisation. Cette méthode constitue un dérivé palynologique de l'ethnobotanique et s'appuie sur la représentation pollinique des végétaux inféodés à l'homme ou à ses pratiques. Ces marqueurs polliniques qui ont fait l'objet de multiples inventaires, essentiellement pour l'Europe du nord (Behre 1981), regroupent les espèces cultivées, mais également les plantes adventices et messicoles ainsi que les espèces rudérales associées aux décombres, aux habitats et plus généralement aux zones humanisées ou à certaines pratiques. Dans tous les cas, et bien qu'il existe des marqueurs fiables et objectifs, la présence d'un seul indice pollinique d'anthropisation ne permet pas de conclure au développement d'activités humaine et seule la concomitance de plusieurs signaux reste significative. Présentées sous la forme de diagrammes synthétiques cumulatifs (diagramme d'anthropisation), les variations de ces taxons-guides permettent d'appréhender sur la longue durée les fluctuations de la pression anthropique en un lieu donné, et permettent des interprétations en termes d'emprise /déprise ou stabilité des activités humaines (Berglund 1969 ; Berglund et RalskaJasiewiczowa 1986).

C'est sur ces bases méthodologiques que les recherches réalisées dans les Pyrénées au cours de ces dernières années ont permis de caractériser les principales étapes et modalités de l'anthropisation du versant nord du massif.

\subsection{Les dynamiques rythmiques de l'anthropisation sur le versant Nord-pyrénéen}

Plusieurs études palynologiques réparties sur l'ensemble du versant nord des Pyrénées permettent de suivre les grandes étapes de l'anthropisation de cette région au cours des sept derniers millénaires (fig.1).

La portée chronologique des séquences utilisées ne permet pas d'aborder les périodes les plus anciennes, en particulier le Néolithique ancien (5700-4500 av. J-C.), durant lequel les premiers signaux d'activités agropastorales apparaissent régionalement. En effet, bien que ceux-ci soient identifiés sur le pourtour languedocien entre la fin du VII ${ }^{e}$ et le $\mathrm{VI}^{\mathrm{e}}$ millénaire av. J.-C. (Jalut 1995, Puertas 1997), aucune étude palynologique réalisée dans les Pyrénées n'a pour l'instant clairement mis en évidence des indices polliniques d'anthropisation aussi précoces, et ce, malgré l'existence de témoignages archéologiques attestant dès cette époque d'une fréquentation du milieu montagnard (Colligà et Vaquer 1995). Ce n'est qu'à la fin de cette période, entre 5200 et 4800 av. J.-C., qu'apparaissent dans les Pyrénées méditerranéennes les premiers signaux polliniques d'activité anthropique. Mais il s'agit principalement d'indices discrets d'activités agraires ou de déforestation touchant les forêts montagnardes (Galop et al. 2003).

En fait, les premiers signes polliniques d'une anthropisation manifeste du milieu montagnard pyrénéen apparaissent à partir du Néolithique moyen (45003500 av. J.-C). Durant cette période, le développement des activités humaines paraît plus affirmé dans les secteurs de piémont, où il s'accompagne de défrichements responsables d'un clarièrage de l'espace forestier, tandis que seuls des indices ponctuels et discrets de déboisement et de fréquentation pastorale sont enregistrés dans les zones d'altitude et dans les vallées montagnardes. En termes de pression anthropique, ces différences enregistrées entre piémont et zone d'altitude pourraient suggérer un déséquilibre dans l'utilisation des zones de basse et de haute montagne. Les zones d'altitude n'auraient été encore vraisemblablement concernées que par des occupations sporadiques ou saisonnières, probablement liées à la mise en place de remues ou d'estivages. Ce décalage s'atténue à partir du Néolithique final où, après une brève phase de déprise, les indices polliniques d'anthropisation reflètent une nouvelle expansion des activités humaines dont le démarrage se situe à la charnière des $\mathrm{IV}^{\mathrm{e}}$ et III $^{\mathrm{e}}$ millénaires av. J.-C. À basse altitude, cette phase se caractérise par la récurrence d'emprises bien marquées, tandis qu'elle paraît plus durable et plus affirmée dans les secteurs d'altitude. Bien que des différences de résolution des analyses polliniques puissent expliquer cette divergence, nous pouvons toutefois émettre l'hypothèse d'une exploitation différente des zones de piémont et d'altitude : les premières auraient été concernées par des pratiques agro-pastorales relativement mobiles (système agro-forestier, Galop et al. 2003) tandis que les zones hautes auraient fait l'objet de fréquentations répétées et pérennes (mise en place de transhumances ?), mais localisées dans des secteurs précis où les potentialités écologiques en termes de ressources herbagères auraient été plus favorables ou plus accessibles.

Quoi qu'il en soit, le développement des activités agro-pastorales, qui s'étend dès lors jusqu'à l'extrémité occidentale de la chaîne, a pour corollaire une modification notable des écosystèmes forestiers. Ainsi, sur le versant nord, l'ouverture progressive des forêts favorise le développement du hêtre au sein des sapinières (Jalut 1984, Galop et Jalut 1994), tandis que les déboisements d'altitude nécessaires à la constitution de pâturages provoquent un abaissement significatif de la limite supérieure des forêts subalpines (Rendu et al. 1995 ; Galop 1998). 
Par la suite, les données pollenanalytiques indiquent peu avant 2000 av. J.-C., au tout début de l'Âge du bronze et vers 1000 av. J.-C., à la transition entre la fin de l'Âge du bronze et l'Âge du fer, l'existence de deux phases distinctes d'expansion. Ces emprises, enregistrées dans la quasi-totalité des séquences polliniques de la chaîne, révèlent des processus régionaux. Deux mille ans avant notre ère, les déboisements s'accentuent et provoquent une ouverture des forêts à toutes les altitudes quoiqu'elle paraisse plus conséquente aux niveaux des grandes vallées et sur le piémont.

C'est à partir de l'Antiquité que les rythmes de l'anthropisation deviennent spatialement plus homogènes. Cette période est caractérisée par un nouvel essor des activités humaines dont les répercussions environnementales se traduisent par des déforestations localisées et apparemment sélectives (principalement le chêne et le sapin) qui pourraient suggérer la mise en place d'un système d'exploitation des forêts compatible avec un souci de rentabilité dans l'utilisation des ressources naturelles.

Si l'on tient compte des données paléoécologiques, le haut Moyen Âge, qui est habituellement considéré comme une période de repli ou de déprise, semble au contraire caractérisé dans les Pyrénées par une relative stabilité voire une expansion des activités agropastorales (Galop 2000a). Sur le piémont, il semble même que des phases d'emprise agraire se soient produites vers les $\mathrm{V}^{\mathrm{e}}$-VII ${ }^{\mathrm{e}}$ siècles (Galop 1998). Ces signes localisés d'augmentation des activités agropastorales rejoignent des indices de croissance suggérés par de rares textes concernant le Midi de la France (Bonnassie 1989).

La phase d'expansion la plus remarquable s'enclenche toutefois entre le IX ${ }^{e}$ et le $\mathrm{X}^{\mathrm{e}}$ siècle, voire dès le VIII $^{e}$ siècle comme cela semble être le cas en Cerdagne. Dans cette région, les données polliniques corroborent les indications fournies par les sources textuelles qui fixent le début de l'expansion agraire dès la fin du $\mathrm{VIII}^{\mathrm{e}}$ siècle et son apogée vers le $\mathrm{XI}^{\mathrm{e}}$ siècle. Bien que les signes d'une véritable explosion des activités agro-pastorales n'apparaissent pas avec la même lisibilité dans toutes les séquences, les dynamiques du couvert forestier sont éloquentes et indiquent une accentuation des déforestations à toutes les altitudes. Le gonflement des terroirs, la mise en place de grands domaines pastoraux monastiques, ainsi que le développement massif des activités métallurgiques (Verna 1994) ont provoqué d'importants déboisements conduisant parfois à une éradication des forêts.

Ainsi que l'on peut le remarquer sur la figure synthétique, cette intensification des activités agrosylvo-pastorales - à l'origine des paysages pyrénéens actuels - est ralentie par une phase de déprise ou de repli centrée sur les $\mathrm{XIV}^{\mathrm{e}}-\mathrm{XV}^{\mathrm{e}}$ siècles. Les enregistrements palynologiques reflètent, ici, les effets de la crise tardo-médiévale qui se sont apparemment accompagnés d'une reforestation. Toutefois, si l'on considère les données, les manifestations de cette crise sont variables. Ainsi, elle n'apparaît pas dans la vallée de l'Aston et plus généralement dans la plupart des vallées métallurgiques de haute Ariège où, en dépit de l'existence de troubles et d'épidémies, le poids économique et l'importance de l'activité métallurgique ont sans doute contribué à masquer ses effets en maintenant notamment une forte pression sur les forêts.

Plus près de nous encore, la reprise postérieure à l'étiage médiéval est clairement mise en évidence dans la plupart des enregistrements polliniques. Cette nouvelle phase d'emprise responsable d'importants défrichements a entraîné une réduction considérable des espaces forestiers conduisant dans certain cas à une véritable crise écologique (Davasse 2000, Galop 1998).

Cette trame chronologique, ainsi que ses interprétations spatiales (Galop 2001 ; Galop et Vaquer sous presse) représentent une étape fondamentale dans la connaissance des mécanismes de l'anthropisation de la montagne pyrénéenne. Elles décrivent les différentes phases d'occupation et d'exploitation de l'espace montagnard en mettant l'accent sur l'existence de seuils rythmant une histoire complexe et caractérisée par d'importantes variabilités spatio-temporelles. Cette trame n'en reste pas moins inachevée et doit être enrichie par des données supplémentaires.

Doit-on pour autant se contenter de cette approche descriptive ? La confrontation interdisciplinaire, avec les archéologues et les historiens en particulier, tend à nous convaincre du contraire. Si en ligne de mire se profile le souci d'une approche intégrée, la confrontation disciplinaire reste difficile. On peut réduire ces difficultés entre palynologie et sciences sociales à trois constats simples : d'une part lorsque l'historien ou l'archéologue parle en termes de quotidienneté ou de dates, nous répondons bien souvent en termes de périodes ou de plages chronologiques plus ou moins étendues et dépendantes de la précision des mesures d'âges obtenues par la méthode du radiocarbone; d'autre part, lorsque ces mêmes spécialistes oeuvrent sur l'histoire, la caractérisation de pratiques socioéconomiques ou des systèmes d'exploitations (élevage, métallurgie, agriculture), nous répondons encore —et certainement par prudence- par les termes vagues « d'anthropisation » ou «d'activités humaines »; enfin, et la liste n'est pas close, face à des données archéologiques ou historiques spatialisés, force est de constater les difficultés que nous rencontrons quant à la spatialisation des informations polliniques. Voilà donc posés aux palynologues à la fois le problème des divergences d'échelles d'analyses, qu'elles soient spatiales et/ou temporelles; et celui plus complexe qui impose une interprétation plus poussée du signal pollinique afin de sortir du flou caractérisant la notion 
d'anthropisation. Pour répondre à ces questions, la palynologie se doit aujourd'hui d'adapter ses méthodes et probablement de modifier certaines tendances dogmatiques.

\section{LE TEMPS « DES PRATIQUES » : DE NOUVELLES PERSPECTIVES DE RECHERCHE}

Les travaux engagés ces dernières années dans les Pyrénées témoignent des initiatives et des avancées méthodologiques effectuées en direction d'une meilleure analyse des manifestations anthropiques. Localement, c'est dans le cadre de deux programmes interdisciplinaires de recherches développés sur la montagne basque (1) que nous avons envisagé la mise en œuvre de nouvelles méthodes qui, pour certaines, restent en cours de validation. Les objectifs n'en restent pas moins affichés : ces démarches paléoenvironnementales doivent permettre d'affiner nos interprétations en matière d'anthropisation en tentant de discriminer les pratiques et les usages au sein d'une information globale fournie par les assemblages polliniques fossiles ; de même qu'il est impératif de maîtriser la signification spatiale de nos données et de lui conférer une résolution temporelle plus précise.

\subsection{Améliorer l'appréciation spatiale du signal pollinique par le croisement des informations : quelques exemples appliqués à la reconstitution des pratiques pastorales}

Si la palynologie est a priori en mesure de répondre à la question de l'échelle temporelle par une systématisation des analyses à haute résolution et par la multiplication des datations par accélérateur réalisées sur des échantillons très réduits (macrorestes végétaux), les problèmes spatiaux et surtout ceux de l'appréciation des pratiques imposent une démarche plus complexe. Elle suppose des analyses intégrées faisant intervenir sur un même enregistrement sédimentaire différents spécialistes ; mais aussi une meilleure interprétation du signal pollinique fondée sur la constitution de référentiels actuels.

Parmi les travaux réalisés au Pays basque, plusieurs exemples illustrent ces nouvelles orientations. Ainsi, dans le registre d'une généralisation des analyses intégrées, dites «multi-proxy », le croisement entre palynologie et géochimie isotopique du plomb a permis de mettre en évidence par le biais des paléopollutions enregistrées dans une tourbière de la haute vallée de Baïgorri, l'histoire locale mais aussi les conséquences environnementales des activités métallurgiques depuis la fin du Néolithique (Galop et Rendu 2001 ; Monna et al. soumis). Les résultats de cette recherche montrent que dès le début de l'Âge du bronze, voir dès le Chalcolithique, les activités métallurgiques ont contribué à l'exploitation du couvert forestier, et peuvent même en être l'origine principale.

Un autre aspect de ces recherches concerne plus spécifiquement la caractérisation et l'étude des activités pastorales. Jusqu'à présent l'approche palynologique de ces pratiques repose sur la prise en compte et l'interprétation de certains indicateurs polliniques représentés par les espèces nitrophiles caractéristiques d'aires de stabulation très fertilisées (Urtica, Chenopodium, Rumex acetosa/acetosella, Cichorioidées), de lieux piétinés (Plantago lanceolata, Plantago major/media...) ou pâturés (Galium, Trifolium, etc...). Cependant, la grande majorité de ces espèces n'est pas exclusivement inféodée aux zones pastorales et elle se rencontre également dans des groupements végétaux postculturaux dominés par des friches herbacées ainsi que dans les prairies de fauche, pour ne citer que ces seuls exemples. Aussi, dans la majorité des cas, ces cortèges polliniques sont-ils seulement interprétés comme le signal témoignant de l'existence d'un milieu anthropisé caractérisé par la présence d'activités agropastorales.

En milieu montagnard, la situation est quelque peu différente : sauf cas extrêmes (montagnes méditerranéennes) où les pratiques agricoles peuvent potentiellement remonter jusqu'à des altitudes voisines de 1900 m, l'élevage est prépondérant et représente le mode d'exploitation principal des espaces d'altitude. Dans de tels contextes, il est clair que les manifestations anthropiques mises en évidence lors des études polliniques pourront être associées sans prendre trop de risques à des activités pastorales. Ainsi, la présence, la régularité des occurrences ainsi que la variation des fréquences relatives de ces taxons sont interprétées comme autant d'indices de l'intensité de la fréquentation, voire de la charge pastorale (Galop 1998 ; Moe et al. 1988 ; Groenman-van Waateringe 1993).

Toutefois, la signification et l'utilisation de ces marqueurs polliniques imposent la prudence. Outre les problèmes liés à la détermination spécifique des grains de pollens (rares sont les cas où il est possible de déterminer avec certitude les types polliniques au niveau de l'espèce), c'est la question de la calibration de ce signal pollinique qui se pose désormais avec insistance.

Ainsi, une étude de la pluie pollinique actuelle, réalisée sur les zones pastorales d'Iraty au pays basque à partir de plus de 80 échantillons de surfaces (Mazier 2001 ; Galop et Mazier, en cours), montre en effet que certains de ces taxons polliniques indicateurs de la fréquentation pastorale sont présents dans la pluie pollinique tout en étant absents de la végétation actuelle. C'est notamment le cas des armoises et des chénopodes.

(1) - Projet Collectif de Recherche 2000-2003 "Paléoenvironnement et dynamiques de l'anthropisation de la montagne basque », coord. D. Galop, SRA Aquitaine, Ministère de la Culture.

- Projet APN du CNRS «Paléoenvironnement et archéologie pastorale : propositions méthodologiques pour une approche intégrée des modalités de l'anthropisation en montagne pyrénéenne du néolithique à l'actuel », coord. D. Galop et C. Rendu, LCE-UMR 6565 CNRS. 
Nous devons par conséquent interpréter des signaux polliniques dont la représentativité spatiale est très variable et reste fonction du mécanisme de pollinisation des espèces végétales (anémogames ou entomogames). À ce titre, les conclusions d'une étude réalisée dans les Alpes du nord sur la composition de la pluie pollinique actuelle dans les zones d'altitude sont éloquentes (Oeggl 1994) : elles distinguent des indicateurs sur-représentés et d'origine extra-régionale (ortie et Poacées) ; des indicateurs sous-représentés et d'origine régionale (armoises, chénopodes, plantains, oseilles...) et enfin des indicateurs qui, beaucoup plus fiables, sont sousreprésentés et d'origine locale (Astéracées, Cichorioidées, Fabacées, Ericacées...).

Le signal pollinique se doit par conséquent d'être confronté à d'autres sources que peuvent fournir d'autres approches paléoécologiques (utilisation des influx polliniques, analyses des macrorestes, étude du signal incendie). Ainsi, certains microfossiles, autres que les grains de pollens ou les spores, sont désormais régulièrement pris en compte lors d'études paléoécologiques (Van Geel 2001 ; Lopez-Saez et al. 1998 ; Galop et al. 2003). Ce sont, en général, des spores d'algues, de cyanobactéries, de champignons, de mousses ou encore des kystes d'origine ontogénique très variée. Si parmi ces micro-fossiles non polliniques (MNP), une grande quantité reste encore à identifier, certains sont utilisés comme indicateurs de l'anthropisation des milieux. Ainsi, les spores de champignons coprophiles appartenant à la famille des Sordariacées (Neurospora sp. ; Cercophora) peuvent être des marqueurs locaux de l'activité pastorale, car souvent associées à la présence de déjections animales sur le site (Van Geel 2001) ; tandis que certaines spores appartenant à des familles de champignons carbonicoles (Coniochaeta, Chaetomium), reflètent l'existence de faciès incendiés ou la présence de charbons sur le site.

Les résultats partiels d'une analyse intégrée réalisée sur la séquence sédimentaire provenant de la tourbière d'Artxilondo à Iraty (Pays basque) confirment l'intérêt de tels croisements (fig. 2). À une analyse pollinique traditionnelle, ont été associées à la fois l'étude du signal incendie (analyse des micro-particules carbonisées) ainsi que celle des MNP. Le synchronisme entre les courbes des types Chaetomium, Cercophora et le signal incendie est remarquable. Chaque augmentation significative de l'influx en particules carbonisées s'accompagne d'une élévation des valeurs de ces deux MNP, ce qui permet de distinguer les incendies locaux d'un bruit de fond régional. $\mathrm{Ce}$ constat confirme également que l'augmentation brutale du signal incendie enregistré par la tourbière correspond à des feux ou des défrichements dans les environs immédiats (Vannière et al. 2001 ; Vannière 2001). On notera également, à partir d'une date antérieure à 2466 cal BC, le synchronisme caractérisant l'apparition des spores de champignons coprophiles (Neurospora et Cercophora) et les premières notations régulières de plantain lancéolé, de plantain majeurs et de céréales (dont la présence semble liée à la puissance des vents ascendants ou bien à des transports épizoïque : Moe et Van der Knapp 1990). Le développement de ces champignons sur la tourbière indique la présence in situ de déjections animales liées à une fréquentation pastorale et révèle le caractère local des pratiques signalées par les taxons polliniques. Les plus anciens vestiges archéologiques témoignant d'une occupation des abords de la tourbière (tumulus Irau IV, Blot 1995) sont par ailleurs contemporains de cette période et datés $3850 \pm 90$ B.P., soit entre 2569 et 2032 av. J.-C. De plus, si l'on considère les variations de ces deux MNP, on constate qu'elles coïncident avec celles des valeurs des indicateurs polliniques du pastoralisme (exemple vers 800 av. J.-C.) ce qui prouverait que dans le cas de l'enregistrement d'Artxilondo, les fluctuations des indicateurs polliniques ont une signification essentiellement locale.

Si cet exemple démontre qu'il est possible d'affiner l'interprétation spatiale d'un signal pollinique témoignant d'une activité pastorale par la mise en place d'une démarche intégrée utilisant d'autres marqueurs paléoécologiques, voire archéologiques, la signification de ce signal du point de vue de son interprétation qualitative (système d'élevage, type de bétail) ou quantitative (pression ou charge pastorale) demeure une question vive.

\subsection{Du pollen aux pratiques pastorales : la piste des référentiels fonctionnels}

La caractérisation des modes d'utilisation du sol représente une piste de recherche classique pour les palynologues. Sur ce sujet, les investigations récentes réalisées en Europe du nord sur la reconstitution des pratiques agraires ont permis de jeter des bases méthodologiques destinées à calibrer le signal pollinique en fonction des systèmes d'exploitations et des paysages (Gaillard et al. 1992 ; Gaillard et Birks 1994 ; Hjelle 1999a et 1999 b ). Le principe de ces recherches qui allient néoécologie et paléoécologie est d'analyser dans des espaces exploités de manière traditionnelle les relations entre type d'utilisation du milieu, végétation et pluie pollinique. En d'autres termes, il s'agit de constituer un référentiel palynologique fonctionnel qui permette l'identification des marqueurs polliniques de l'activité humaine et de distinguer les différentes pratiques. Les données palynologiques fossiles peuvent par la suite être confrontées à ce référentiel en recherchant notamment, les correspondances avec les analogues actuels les plus proches (Räsänen 2001 ; Gaillard et Birks 1994). Les résultats très prometteurs avancés par ces recherches conservent cependant une valeur régionale difficilement transposable aux zones méridionales de l'Europe.

Un travail similaire s'est donc engagé à Iraty, dans les Pyrénées basque, afin d'optimiser les possibilités d'interprétation des données palynologiques fossiles recueillies dans plusieurs tourbières situées au cœur de cette zone pastorale (Galop et Rendu 2001 ; Mazier 2001). Un référentiel palynologique fonctionnel ciblé sur les pratiques pastorales est en cours de constitution à 


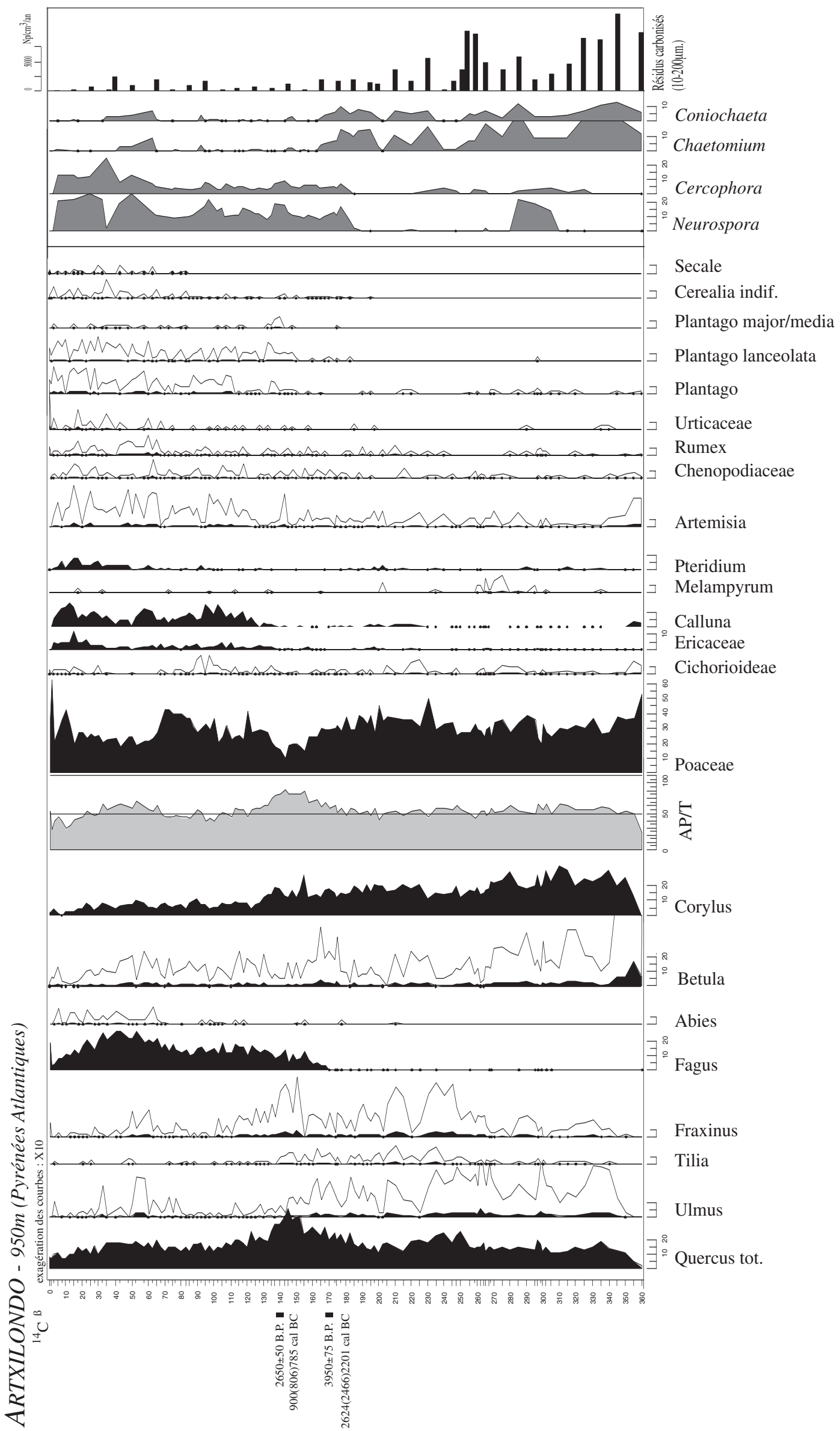

Fig. 2 - Diagramme «multi-proxy » simplifié de la séquence d'Artxilondo (Iraty, Pays basque).

Analyse pollinique : D. Galop ; Analyse des MNP : J.A. Lopez-Saez ; Analyse des résidus carbonisés : B. Vannière. 
l'heure actuelle et son élaboration est largement facilitée par l'existence locale d'une intense activité pastorale qui a conservé selon les secteurs un caractère traditionnel. La pratique de l'estivage, le maintien des parcours, le pâturage sous forêt, ainsi que l'utilisation des couloirs de traite indispensables aux productions fromagères ont favorisé la pérennisation des faciès végétaux directement inféodés à ces pratiques ou aux structures qui leur sont associées. Dans ce contexte, près de quatre-vingts placettes représentatives et situées à proximité des tourbières afin de faciliter les possibilités de transferts (Gaillard et al. 1992 ; Hjelle 1998) sont en cours d'étude. Sur chacune des placettes, une analyse de la pluie pollinique actuelle est couplée à une étude de la végétation (2), tandis que l'ensemble des paramètres environnementaux (pédologiques, topographiques) et anthropiques (charge pastorale, nature du cheptel, proximité des structures pastorales) sont quantifiés. Différentes analyses statistiques sont employées afin de traiter ces données. Une première analyse (méthode des scatters plots et indices de Davis, 1984) permet d'estimer la représentativité pollinique de certaines taxons, c'est-àdire en observant s'ils sont correctement, sur- ou sousreprésentés, par rapport à la végétation environnante.
Ceci a permis d'identifier les types polliniques indiquant la présence locale d'une plante. Une deuxième analyse permet quant à elle la confrontation de l'ensemble des informations recueillies. Elle s'effectue au moyen d'une analyse canonique des correspondances (ACC) qui permet de mesurer l'effet des différents facteurs sur la structure des spectres polliniques : l'objectif étant de pouvoir relier les données polliniques aux variables pastorales (charge, cheptel etc...).

Les premiers résultats de ce travail concernent principalement le comportement pollinique de certains taxons herbacés dans des contextes fortement pâturés. Cette étude préalable permet de préciser la signification réelle de certains marqueurs polliniques du pastoralisme (tableau 1). D'autre part, les premières ordinations sous contrainte (ACC) réalisées sur la base de quelques échantillons ont permis d'identifier les variables responsables de la dispersion des relevés polliniques : parmi celles-ci, la variable « pression de pâture » est très significative (Mazier 2001). Elle conditionne la présence des taxons tels qu'orties, cirses, plantain majeur, oseille, Cichorioidées, Scrophulariacées et Renonculacées.

\begin{tabular}{|c|c|c|}
\hline Taxons polliniques & Espèces floristiques & $\begin{array}{l}\text { Représentation } \\
\text { pollinique }\end{array}$ \\
\hline Artemisia & & $\mathrm{O}$ \\
\hline Aster type bellis & Bellis perennis & A \\
\hline Asteraceae & $\begin{array}{l}\text { Achillea millefolium, Asteraceae sp., Carduus defloratus, } \\
\text { Chrisanthemum leucanthemum, Serratula tinctoria }\end{array}$ & A \\
\hline Cichorioideae & $\begin{array}{l}\text { Crepis biennis, Hieracium pilosella, Hypochoeris radicata, } \\
\text { Leondoton hispidus, Senecio sp., Taraxacum officinale, Tragopogon } \\
\text { pratensis }\end{array}$ & A \\
\hline Brassicaceae & $\begin{array}{l}\text { Arabis hirsuta, Capsella bursa-pastoralis, Sisymbrium officinale, } \\
\text { Brassicaceae sp. }\end{array}$ & $\mathrm{O}$ \\
\hline Calluna & Calluna vulgaris & A \\
\hline Cirsium type & $\begin{array}{l}\text { Cirsium acaule, Cirsium arvense, Cirsium eriophorum richterianum, } \\
\text { Cirsium lanceanum, Cirsium palustre, Cirsium uluginosum }\end{array}$ & $\mathrm{U}$ \\
\hline Ericaceae & Erica tetralix, Erica vagans, Vaccinium myrtillus & A \\
\hline Fabaceae & $\begin{array}{l}\text { Genista hispanica occidentalis, Lathyrus pratensis, Medicago } \\
\text { lupulina }\end{array}$ & $\mathrm{U}$ \\
\hline Galium type & $\begin{array}{l}\text { Galium anisophyllon, Galium cruciata, Galium elongatum, Galium } \\
\text { hercynicum, Galium verum }\end{array}$ & A \\
\hline Labiateae & $\begin{array}{l}\text { Clinopodium vulgare, Lamium maculatum, Lamium purpureum, } \\
\text { Prunella laciniata, Prunella vulgaris, Stachys officinalis, Stachys } \\
\text { sylvatica }\end{array}$ & $\mathrm{U}$ \\
\hline Plantago sp. & & $\mathrm{X}$ \\
\hline Plantago lanceolata & Plantago lanceolata & $\mathrm{X}$ \\
\hline Plantago major/media & Plantago major, Plantago media & $\mathrm{U}$ \\
\hline Poaceae & Toutes les Poacées & $\mathrm{O}$ \\
\hline Ranunculaceae & $\begin{array}{l}\text { Aconitum napellus, Hepatica nobilis, Ranunculaceae sp., Ranunculus } \\
\text { sardous, Ranunculus bulbosus, Ranunculus repens }\end{array}$ & A \\
\hline Rumex & Rumex obtusifolius & $\mathrm{O}$ \\
\hline Rumex acetosa/acetosella & Rumex acetosella & $\mathrm{O}$ \\
\hline Scrophulariaceae & $\begin{array}{l}\text { Digitalis purpurea, Euphrasia sp., Scrophularia nodosa, Veronica } \\
\text { arvensis, veronica chamaedrys, Veronica serpyllifolia }\end{array}$ & $\mathrm{U}$ \\
\hline Urticaceae & Urtica dioica & $\mathrm{U}$ \\
\hline
\end{tabular}

Tabl. 1 - Représentation pollinique des taxons polliniques et des plantes associées (d'après Mazier 2001). A = bonne corrélation ; $\mathrm{U}=$ sous-représentation pollinique $\mathrm{O}=$ sur représentation pollinique $; \mathrm{X}=$ pas d'indication.

(2) L'analyse de la végétation est réalisée à partir d'une approche synusiale intégrée. Cette approche (Gillet 1986 ; Foucault 1986 ; Gillet et Gallandat 1996) représente un perfectionnement de la phytosociologie. Elle permet de rendre compte des différents niveaux de complexité de l'organisation des écosystèmes et décrit la complexité des structures végétales. 
Toutefois, il ressort également que ces résultats possèdent une valeur essentiellement locale et qu'il serait dangereux de les transposer directement à d'autres secteurs d'étude. Le transfert de ces référentiels polliniques aux données fossiles reste assujetti à l'achèvement des traitements statistiques et représente par conséquent la prochaine étape de cette recherche.

\section{CONCLUSION}

"Le dialogue entre sciences de la nature et de la société relève certes d'échelles de temps et d'espaces hétérogènes, mais la difficulté ne doit pas occulter l'intérêt d'études conjointes des milieux naturels et des espaces sociaux pour reconstituer les héritages et les liens entre dynamiques naturelles et sociales »(Moriceau et Schneider 2002). Ces lignes reprises dans l'introduction d'un volume récent de la revue Histoire et Sociétés Rurales qui ouvrait pour la première fois ses colonnes à des études polliniques (Barbier et al. 2002), sont symptomatiques des entraves caractérisant l'intégration des sources dans le cadre d'études interdisciplinaires. Les expériences menées sur le terrain pyrénéen l'avaient déjà largement souligné (Rendu et al. 1999 ; Rendu 2003) et de nouveaux programmes de recherches sont aujourd'hui le support au développement de nouvelles méthodologies dans le domaine des disciplines du paléoenvironnement et de la palynologie en particulier. Certaines, visant notamment à la réduction des échelles temporelles ou bien à une meilleure définition spatiale du signal pollinique, semblent opportunes et fécondes ; tandis que la mise en place d'une démarche fondée sur l'utilisation de référentiels polliniques fonctionnels n'est pas encore suffisamment aboutie pour que l'on puisse tirer des conclusions objectives. Malgré cela, les résultats provisoires acquis sur la montagne basque démontrent la nécessité de poursuivre et d'élargir cette démarche. Dans cette perspective où l'acquisition de référentiels actuels devient impérieuse (tant au niveau des assemblages polliniques, que des microfossiles non polliniques, ou du signal incendie), l'ouverture en direction d'autres disciplines paléoécologiques, mais surtout la collaboration avec les écologues et enfin, un retour sur la réalité du terrain paraissent incontournables. À ce stade, on ne peut que rappeler la formule du biogéographe Henri Gaussen qui en 1936, dans un article polémique au sujet des résultats proposés par la palynologie (discipline naissante à cette époque), concluait son article par : «Un peu d'air, un peu de vues générales, ne regardons pas le monde uniquement avec un microscope »(Gaussen 1933).

Les pistes de recherche que nous proposons ne sont pas fermées et ne se cantonnent aux montagnes pyrénéennes : en témoignent plusieurs travaux en cours dans les Alpes (Court-Picon, ce volume) et le Massif central (Miras, Thèse en cours) qui prennent aujourd'hui des directions similaires. Il faut maintenant souhaiter qu'une réflexion globale sur le sujet —n'impliquant pas seulement la communauté des palynologues - puisse s'instaurer afin de confronter nos expériences respectives.

\section{BIBLIOGRAPHIE :}

Aubert 2001 : AUBERT (S.), Limite supérieure de la forêt, climat et anthropisation : dynamique tardiglaciaire et holocène de la végétation dans la vallée du Marcadau (Hautes-Pyrénées, France), Thèse de Doctorat, Université Toulouse III, 2001, 365 p. et diagrammes.

Barbier et al. 2002 : BARBIER (D.), VISSET (L.) et BURNOUF (J.),Une source pollinique et son exploitation. A propos de la tourbière de Glatinié (Mayenne), Histoire et Sociétés Rurales, 18, 2002, p. 137-158.

Behre 1981 : BEHRE (K. E.), The interpretation of anthropogenic indicators in pollen diagrams, Pollen et Spores, 23, 1981, p. 225-245.

Belet 2001 : BELET (J.-M.), Végétation tardiglaciaire et holocène de la limite supérieure de la forêt dans les Pyrénées centrales : l'exemple du vallon d'Estibère (Hautes-Pyrénées, France), Thèse de Doctorat, Université Toulouse III, 2001, 241 p. et diagrammes.

Berglund 1969 : BERGLUND (B. E.), Vegetation and human influence in south scandinavia during prehistoric times, Oikos Suppl., 12,1969, p. 9-28.

Berglund et Ralska-Jasiewiczowa 1986 : BERGLUND (B. E.) et RALSKA-JASIEWICZOWA (M.), Pollen analysis and pollen diagrams, in : BERGLUND (B. E.) éd., Handbook of Holocene Palaeoecology and Palaeohydrology, John Wiley and Sons Ltd., 1986, p. 455484.

Blot 1995 : BLOT (J.), Contribution à l'étude des cercles de pierres en pays basque de France, Bulletin de la S.P.F., 92, 4, 1995, p. 525-548.

Bonnassie 1989 : BONNASSIE (P.), La croissance agricole du Haut Moyen Age dans la Gaule du midi et le nord-est de la péninsule ibérique, La croissance agricole du haut Moyen Âge, Flaran, 10, 1989, p. 13-35.

Colliga et Vaquer 1995 : COLLIGA (M.) et VAQUER (J.), El poblament dels Pirineus a l'Holocè, del Mesolitic a l'edat del Bronze. Muntanyes i poblacio. El passat dels Pirineus des d'una perspectiva multidisciplinària, Centre de trobada de les cultures Pirinenques, Andorra la Vella, 1995, p. 3572 .

Davasse 2000 : DAVASSE (B.), Forêts, charbonniers et paysans dans les Pyrénées de l'est du Moyen Âge à nos jours. Une approche géographique de l'histoire de l'environnement, Geode, Université de Toulouse le Mirail, 2000, 287 p.

Davis 1984 : DAVIS (O. K.), Pollen frequencies reflect vegetation patterns in a Great Bassin (USA) mountain range. Review of Palaeobotany and Palynology, 40, 1984, p. 295-315.

Foucault 1986 : FOUCAULT de (B.), La phytosociologie sigmatiste : une morpho-physique. Thèse, Lille, 1986, $147 \mathrm{p}$. 
Gaillard et al. 1992 : GAILLARD (M.-J.), BIRKS (H. J. B.), EMANUELSSON (U.) et BERGLUND (B. E.), Modern pollen/land-use relationships as an aid in the reconstruction of past land-uses and cultural landscapes : an example from south Sweden, Vegetation History and Archaeobotany, 1, 1992, p. 3-17.

Gaillard et Birks 1994 : GAILLARD (M.-J.) et BIRKS (H. J. B.), Application of modern pollen/land-use relationships to the interpretation of pollen diagrams - reconstructions of land-use history in south sweden , 3000-0 BP., Review of Palaeobotany and Palynology, 82, 1994, p. 47-73.

Galop 1992 : GALOP (D.), Approche palynologique de l'impact de la métallurgie au bois sur les forêts de la vallée du Quioulès (Pyrénées ariégeoises), in: METAILIE (J.-P.) éd., Proto-industries et histoire des forêts, Les cahiers de l'ISARD, 3, 1992, 257-270.

Galop 1998 : GALOP (D.), La forêt, l'homme et le troupeau dans les Pyrénées. 6000 ans d'histoire de l'environnement entre Garonne et Méditerranée. GEODE-LETFRA.M.ESPA, Toulouse, 1998, 285 p.

Galop 1999 : GALOP (D.), Le parchemin et le pollen : la Cerdagne médiévale de l'archive écrite à l'archive naturelle. Les sociétés méridionales à l'âge féodal, Hommage à Pierre Bonnassie, CNRS. Presse Universitaire du Mirail, 1999, p. 35-43.

Galop 2000a : GALOP (D.), La croissance médiévale sur le versant nord des Pyrénées à partir des données palynologiques, in: BERTHE (M.) et CURSENTE (B.) éd., Villages Pyrénéens. Morphogenèse d'un habitat de montagne, CNRS, UTM, 2000, p. 45-54.

Galop 2000b : GALOP (D.), Propagation des activités agropastorales sur le versant nord-pyrénéen entre le $\mathrm{VI}^{\mathrm{e}}$ et le III ${ }^{\mathrm{e}}$ millénaire av. J.-C. : l'apport de la palynologie, Rencontres Méridionales de Préhistoire récente, $3^{e}$ session, Archives d'Ecologie Préhistoriques, 2000, p. 101-108.

Galop 2001 : D GALOP (D.), Les apports de la palynologie à l'histoire rurale : l'exemple de la longue durée des activités agro-pastorales pyrénéennes, in : GUILAINE (J.) dir., $L a$ très longue durée, Etudes Rurales, 153-154, 2001, p. 127 138

Galop et Jalut 1994 : GALOP (D.) et JALUT (G.) Differential human impact and vegetation history in two adjacent Pyrenean valleys in the Ariège basin, southern France, from $3000 \mathrm{BP}$ to the present, Vegetation History and Archeobotany, 3, 1994, p. 225-244.

Galop et Rendu 2001 : GALOP (D.) et RENDU (C.), Paléoenvironnement et archéologie pastorale. Propositions méthodologiques pour une approche intégrée des modalités de l'anthropisation en haute montagne pyrénéenne du Néolithique à l'actuel, Projet APN du CNRS, Labo. Chrono-Eco, Besançon, 2001, 23 p.

Galop et al. 2001b : GALOP (D.), TUAL (M.), MONNA (F.), DOMINIK (J.), BEYRIE (A.) et MAREMBERT (F.), Cinq millénaires de métallurgie en montagne basque. Les apports d'une démarche intégrée alliant palynologie et géochimie isotopique du plomb. Sud-Ouest Européen, 11, 2001, p. 3-15.
Galop et al. 2003 : GALOP(D.), VANNIERE (B.) et LOPEZSAEZ (J.-A.), Des abattis-brûlis néolithiques au système agro-pastoral pyrénéen actuel. Mise en évidence pluridisciplinaire de l'évolution du système agraire dans une vallée du piémont nord-pyrénéen entre le Néolithique ancien et l'Antiquité tardive, in: Pirineus $i$ veïns al $3 r$ mil.leni AC. De la fi del Neolític a l'Edat del Bronze entre l'Ebre $i$ la Garona, $\mathrm{XII}^{\mathrm{e}}$ Colloque international d'archéologie de Puigcerda. Institut d'Estudis Ceretans, 2003, p. 261-274.

Galop et Vaquer sous presse : GALOP (D.) et VAQUER (J.), Regards croisés sur les premiers indices de l'anthropisation en domaine pyrénéen, in : Richard (H.) éd., Premières traces de l'impact de l'homme sur la végétation Holocène, Coll. Annales littéraires, série Environnement, Sociétés et Archéologie, Presses Universitaires de Franche-Comté, sous presse, $21 \mathrm{p}$.

Gaussen 1933 : GAUSSEN (H.), A propos de la période xérothermique. Bulletin de la société d'Histoire naturelle de Toulouse, 68, fasc.2, 1933, p. 342-353.

Gillet 1986 : GILLET (F.), Analyse concrète et théorique des relations à différents niveaux de perception phytoécologique entre végétation forestière et géomorphologie dans le Jura nord-occidental. Colloque phytosociologique, 13, 1986, p. 101-131.

Gillet et Gallandrat 1996 : GILLET (F.) et GALLANDRAT (J.-D.), Integrated synusial phytosociology : some notes on a new multiscalar approach to vegetation analysis, Journal of Vegetation Science, 7, 1996, p. 13-18.

Groenman-van-Waeteringe 1993 : GROENMAN-VANWAETERINGE (W.), The effect of grazing on the pollen production of grasses, Vegetation History and Archaeobotany, 2, 1993. 157-162.

Guilaine 1995 : GUILAINE (J.), Introduction au colloque. Culturi i medi de la prehistoria a l'edat mitjana, Actes du $\mathrm{X}^{\mathrm{e}}$ Col.loqui internacional d'arqueologia de Puigcerda, Institut d'Estudis Ceretans, 1995, p. 61-63.

Hjelle 1998 : HJELLE (K. L.), Herb pollen representation in surface moss samples from mown meadows and pastures in western Norway, Vegetation History and Archaeobotany, 7, fasc. 2, 1998, p. 79-96.

Hjelle 1999a : HJELLE (K. L.), Use of modern pollen samples and estimated pollen representation factors as aids in the interpretation of cultural activity in local pollen diagrams, Norwegian Archaeological Review, 32, fasc. 1, 1999, p. 1939.

Hjelle 1999b : HJELLE (K. L.), Modern pollen assemblages from mown and grazed vegetation types in western Norway, Review of Palaeobotany and Palynology, 107, 1999, p. 55-81.

Jalut 1974 : JALUT (G.), Evolution de la végétation et variations climatiques durant les quinze derniers millénaires dans l'extrémité orientale des Pyrénées, Thèse d'Etat, Université Toulouse III, 1974, 176 p. 
Jalut 1984 : JALUT (G.), L'action de l'homme sur la forêt montagnarde dans les Pyrénées ariègeoises et orientales depuis $4000 \mathrm{BP}$ d'après l'analyse pollinique. Actes $d u$ 106ème Cong. Nat. des sociétés savantes, sect. Géographie, Perpignan 1981 (Paris 1984), 1984, p. 163 172 .

Jalut 1995 : JALUT (G.), Analyse pollinique de sédiments holocènes de l'étang de Capestang (Hérault), in : GUILAINE (J.) dir., Temps et espace dans le bassin de l'Aude $d u$ Néolithique à l'âge $d u$ Fer, Centre d'Anthropologie, Toulouse, 1995, p. 293-303.

Kvamme 1988 : KVAMME (M.), Pollenanalytical studies of mountain summer farming in western Norway, in : BIRKS (H.H.), BIRKS (H. J. B.), KALAND (P. E.) et MOE (D.) éd., The cultural landscape. Past, Present and Future, Cambridge University Press, 1988, p. 349-367.

López-Sáez et al. 1998: LOPEZ-SAEZ (J.A.), VAN GEEL (B.), FARBOS-TEXIER (S.), et DIOT (M.-F.), Remarques paléoécologiques à propos de quelques palynomorphes non-polliniques provenant de sédiments quaternaires en France, Revue de Paléobiologie de Genève, 17, 2, 1998, p. 445-459.

Mazier 2001 : MAZIER (F.), Validation des signatures polliniques du pastoralisme en moyenne montagne pyrénéenne. Etude des relations végétation/pluie pollinique actuelle et pratiques pastorales, Diplômes d'Etudes Approfondies, Université de Franche-Comté, Besançon : 2001. $32 \mathrm{p}$.

Moe et al. 1988 : MOE (D.), INDRELID (S.) et FASTELAND (A.), The Halne area, Hardangervidda. Use of a high mountain during 5000 years - An interdisciplinary case study. in : BIRKS (H.H.), BIRKS (H. J. B.) ; KALAND (P. E.) et $\operatorname{MOE}$ (D.) éd., The cultural landscape. Past, Present and Future, Cambridge University Press, 1988, p. 429-444.

Moe et Van der Knapp 1990 : MOE (D.) et VAN DER KNAPP (W.O.), Transhumance in mountain areas : additional interpretation of three pollen diagrams from Norway, Portugal and Switzerland, in : MOE (D.) et HICKS (S.) éd., Impact of prehistoric and medieval man on the vegetation : man at the forest limit, PACT, 31, 1990, p. 91-102.

Monna et al. soumis : MONNA (F.), GALOP (D.), CAROZZA (L.), TUAL (M.), BEYRIE (A.), MAREMBERT (F.) et DOMINIK (J.), Impact of local early metalworking in the Basque country pointed out by geochemical and pollen records in minerotrophic peatlands, Journal of Geochemical Exploration, soumis.

Moriceau et Schneider 2002 : MORICEAU (J.-M.) et SCHNEIDER (L.), La mobilité : une notion à définir et à relativiser, Histoire et Sociétés Rurales, 18, 2002, p. 7-10.

Oeggl 1994 : OEGGL (K.), The palynological record of human impact on highland zone ecosystems, in : BIAGI (P.) et NANDRIS (J.) éd., Highland zone exploitation in southern Europe, Monographie di Natura Bresciana, 20, 1994, p.107-122.
Puertas 1997 : PUERTAS (O.), Evolution de la végétation depuis le Dryas récent dans la plaine littorale de Montpellier (Hérault, France) à partir de l'analyse pollinique. Dynamique naturelle et anthropisation $d u$ milieu, Thèse de Doctorat, Université de Franche-Comté, 1997, 337 p.

Räsänen 2001 : RÄSÄNEN (S.), Tracing and interpreting finescale human impact in northern Fennoscandia with the aid of modern pollen analogues, Vegetation History and Archaeobotany, 10, 2001, p. 211-218.

Reille 1990 : REILLE (M.), Recherches pollenanalytiques dans l'extrémité orientale des Pyrénées : données nouvelles de la fin du glaciaire à l'actuel, Ecologia Mediterranea, XVI, 1990, p. 317-357.

Reille et al. 1994 : REILLE (M.) et ANDRIEU (V.), Vegetation history and human action in Ariège (Pyrénées, France), Dissertationes Botanicae, 234, 1994, 413-422.

Rendu 2003 : RENDU (C.), La montagne d'Enveig. Une estive pyrénéenne dans la longue durée, Perpignan, éd. du Trabucaire, 2003, $606 \mathrm{p}$.

Rendu et al. 1995 : RENDU (C.), CAMPMAJO (P.), DAVASSE (B.) et GALOP (D.), Habitat, environnement et systèmes pastoraux en montagne : acquis et perspectives de recherches à partir de l'étude du territoire d'Enveig. Cultures $i$ medi de la prehistòria a l'edat mitjana. $\mathrm{X}^{\mathrm{e}}$ Col.loqui internacional d'Arquéologia de Puigcerda. Homenatge a Jean Guilaine, Institut d'Estudis Ceretans, 1995, p. 661-673.

Rendu et al. 1999 : RENDU (C.), DAVASSE (B.), GALOP (D.), EVIN (J.) et FONTUGNE (M.), Archéologie et Environnement en haute montagne. L'apport des datations radiocarbone, in : EVIN (J.), OBERLIN (C.), DAUGAS (J.-P.) et SALLES (J.-F.) éd., " C14 et archéologie ", Mémoire de la S.P.F., t. 26 et supplément 1999 de la Revue d'Archéométrie, 1999, p. 411-417.

Van Geel 2001 : VAN GEEL (B.), Non-pollen palynomorphs, in : SMOL (J.P.), BIRKS (H.J.B.) et LAST (W.M.) éd., Tracking environmental change using lake sediments. Vol 3, Kluwer academic Publishers, Dordrecht, The Netherlands, 2001, p. 99-119.

Vannière 2001 : VANNIERE (B.), Feu, agro-pastoralisme et dynamiques environnementales en France durant l'Holocène. Analyse du signal incendie, approches sédimentologiques et étude de cas en Berry, Pyrénées et Franche-Comté. Thèse de l'Institut National Agronomique de Paris-Grignon, 2001, 329 p.

Vannière et al. 2001 : VANNIERE (B.), GALOP (D.), RENDU (C.) et DAVASSE (B.), Feu et pratiques agropastorales dans les Pyrénées-Orientales : le cas de la montagne d'Enveitg (Cerdagne, P.-O., France), Sud-Ouest Européen, 11, 2001, p. 29-42.

Verna 1994: VERNA (C.), Le temps des moulines. Le fer et son exploitation du Comté de Foix à la Vicomté de Béarn (Fin du XII siècle - fin $d u X V^{e}$ siècle). Thèse de Doctorat, Université de Paris I, 2 vol., 1994, 495 p. et 196 p. 